\title{
Reflexiones sobre la convivencia escolar y la práctica del Mindfulness
}

\author{
Reflections about scholar coexistence and the practice of Mindfulness
}

\author{
Magaly Calderón Uribe ${ }^{a}$, Diana Melissa Otálora Gallego ${ }^{b}$, \\ Sandra Patricia Guerra Báez ${ }^{c}$, Efrain David Medina Giraldo ${ }^{d}$
}

\author{
${ }^{a}$ Corporación Universitaria Minuto de Dios, Colombia \\ Correo electrónico: mcalderon@uniminuto.edu.co \\ ${ }^{\mathrm{b}}$ Corporación Universitaria Minuto de Dios, Colombia \\ Correo electrónico: dotaloragal@uniminuto.edu.co \\ ${ }^{c}$ Fundación Universitaria Konrad Lorenz

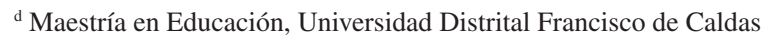

\begin{abstract}
RESUMEN
El Mindfulness es una práctica en auge desde hace unas décadas a nivel mundial, por lo que el presente artículo se propone hacer una reflexión acerca del Mindfulness como una herramienta que prepara a los estudiantes para el desarrollo de competencias ciudadanas y, por ende, para la convivencia escolar. Para lograr dicho objetivo, en un primer momento se definirá el concepto de convivencia para el contexto colombiano y las leyes desarrolladas en relación con ésta, así como, algunas estrategias implementadas para fortalecerla. En segundo lugar, se abordará la práctica de Mindfulness, su definición y etapas e incursión en el ámbito escolar. Se concluye realizando una reflexión sobre el aporte que ésta puede hacer no sólo a la convivencia escolar en general, sino al desarrollo de las competencias ciudadanas propuestas como una estrategia de intervención del gobierno para formar ciudadanos más conscientes, relaciones más sanas y entornos más seguros.
\end{abstract}

Palabras clave: atención plena, competencias ciudadanas, comportamiento, consciencia plena, relaciones escolares

\begin{abstract}
Worldwide, since a few decades, Mindfulness has been a practice in growth. This article proposes a reflection about mindfulness as a tool that prepares students for the development of citizen abilities and, thus, for school coexistence. In order to reach that objective, first the concept of coexistence will be defined within the Colombian context and the laws regarding it, as well as the strategies implemented in order to strengthen it. Secondly, we will address the practice of mindfulness, it's definition, stages and entrance in school context. As a conclusion, a reflection about mindfulness contribution to scholar coexistence and citizen abilities will be carried out, proposing it as a strategy of government intervention to educate more conscious citizens, healthy relationships and safe environments.
\end{abstract}

Key words: full Attention, citizen abilities, behavior, full consciousness, school relations 


\section{INTRODUCCIÓN}

Colombia es un país que en las últimas décadas ha vivido diferentes procesos sociopolíticos relevantes (reforma constitucional, proceso de paz, etc.), los cuales han impactado no sólo en las diferentes instituciones sino en cada sujeto inmerso en ellas. En la educación y, específicamente, en la convivencia, a partir del 2013 con la Ley 1620, se reglamentó el Sistema Nacional de Convivencia, el cual intentó establecer mecanismos para promover, prevenir y ayudar a mejorar el clima escolar en las organizaciones y disminuir las acciones que atentan contra los derechos humanos, sexuales y reproductivos (MEN, 2013). Esta ley ha llevado a que las instituciones educativas le den relevancia a conocer y adoptar diferentes estrategias que ayuden a abordar las problemáticas relacionadas con la convivencia.

La convivencia es un término complejo y difícil de definir desde una sola perspectiva, puesto que no sólo son diferentes las disciplinas que lo abordan, sino también el contexto influye en dicha percepción. La definición del concepto de convivencia puede variar en nuestro país de acuerdo a las condiciones socioeconómicas, psicológicas y educativas, pues no es lo mismo la percepción que puede tener un estudiante de un colegio privado a uno de un colegio público. Inclusive, la percepción de un estudiante y otro del mismo colegio puede variar, pues sus experiencias y aprendizajes en su entorno social y familiar probablemente son distintos. De acuerdo con Herrera y Silas (2017), la convivencia se puede comprender como aquel proceso en el cual se ve involucrado el sujeto con los otros, en el que se ponen a prueba sus valores, percepciones, experiencias y aprendizajes adquiridos en su vida, visualizando la convivencia como un reflejo de la cultura.

Marrugo, Gutiérrez, Concepción y Concepción (2016) afirman que la convivencia es interactuar y compartir aspectos comunes de manera activa con otras personas en un momento y espacio determinado. Esto permite el desarrollo de valores y habilidades, las cuales son la base del futuro ciudadano, un proceso que generalmente se da en el contexto escolar (Megías, 2011).

Sin embargo, aterrizar la convivencia en el ámbito escolar significa comprenderla desde diferentes perspectivas. Esto quiere decir que debemos conocer las formas como se está abordando y concibiendo dentro de la comunidad educativa. Ejemplo de esto son los estudios de Enrique Chaux, uno de los pioneros en abordar la convivencia en Colombia, quien aproximadamente desde el año 2000 ha analizado los conflictos y agresiones entre niños y adolescentes y la violencia en los colegios de Bogotá. Este autor se ha enfocado en profundizar, con sus investigaciones y proyectos, temas como la educación de la convivencia, la intimidación escolar (bullying) y la prevención de la violencia.

Otra de las formas en cómo se está abordando la convivencia en Colombia son las competencias ciudadanas; las cuales desde el 2004 surgieron con la intención de transformar las cátedras asociadas a la instrucción cívica tradicional en propuestas pedagógicas transversales que promuevan las habilidades, actitudes y conocimientos necesarios para lograr una sana convivencia escolar (MEN, 2011). Estas propuestas revisadas dan cuenta del énfasis que, en la mayoría de estrategias, se le ha dado a la convivencia, explicando las reacciones del estudiante a partir de su entorno y colocando la responsabilidad solo en el contexto, dejando de lado el aspecto introspectivo que enfatiza en el fortalecimiento de las habilidades del sujeto como la autorregulación, el autocontrol, la conciencia de sí mismo y la autogestión, entre otras. A partir de ese vacío cobra relevancia el Mindfulness. 
El Mindfulness es una práctica en auge desde hace unas décadas a nivel mundial y de reciente aparición en el contexto colombiano, la cual es un proceso de intervención basado en la atención plena, en el aquí y ahora. Kabat-Zin, uno de los precursores de esta práctica, la define como una conciencia sostenida, deliberada y sin juicios de valor que se desarrolla prestando atención concreta al momento presente (2013). La práctica del Mindfulness, combinada con técnicas utilizadas por la psicología como la observación, entrenamiento en habilidades, registros, control y manejo de emociones, etc., han contribuido en el tratamiento de diversos problemas tanto físicos como mentales aportando a mejorar el bienestar y la calidad de vida de las personas (Hervás, Cebolla \& Soler, 2016).

Específicamente, en los colegios el Mindfulness está siendo utilizado para resolver conflictos, fortalecer habilidades sociales, mejorar procesos académicos y problemas de ansiedad. En Colombia, aunque la práctica sigue siendo reciente, ya existen algunas investigaciones relacionadas con el Mindfulness, por ejemplo, el estudio de Alajmo y Jiménez (2016) que a través de un programa basado en Mindfulness disminuyeron la ansiedad de los estudiantes en niveles físicos, además de mejorar la capacidad para regularse y mejorar los pensamientos distractores. En otro estudio enfocado en estudiantes universitarios con problemas de atención, desarrollado por Barragán, Lewis y Palacio (2007), se encontró que éste método generó cambios significativos en la autopercepción de los subprocesos atencionales y en los indicadores de atención del WAIS (Escala de inteligencia de Wechsler para adultos).

Basado en lo anterior, el presente artículo abordará el concepto de convivencia, las leyes desarrolladas en relación con ésta y algunas estrategias implementadas para fortalecerla. Asimismo, se define el Mindfulness, sus etapas e incursión en el ámbito escolar. Para cerrar con la reflexión sobre el aporte que ésta técnica puede hacer no sólo a la convivencia escolar en general, sino al desarrollo de las competencias ciudadanas.

\section{CONVIVENCIA ESCOLAR}

Convivir es un valor agregado de autonomía que se construye día a día, con plena conciencia del respeto por uno mismo, por los otros y por el ambiente. Para Maturana (2002), la convivencia surge como consecuencia de las interrelaciones entre las personas y el medio en el cual están inmersas, es decir, son experiencias que surgen de diferentes contextos relacionados con cada sujeto. Por nuestra misma naturaleza, somos individuos que se relacionan constantemente con otros. De esa interacción, surgen aprendizajes que son puestos a prueba en los diferentes ámbitos de nuestras vidas a través de la convivencia con los otros. En este sentido, la convivencia es concebida como el reflejo de la cultura que se desarrolla en la vida cotidiana e involucra el modo de ser de cada persona en interrelación con los otros, donde cada sujeto pone en práctica sus valores, creencias, percepciones, reflexiones y conocimiento adquirido, lo que conlleva a mejores formas de vida en sociedad (Herrera \& Silas, 2017).

La convivencia es entonces un término difícil de definir desde una perspectiva unilateral, pues son varios los puntos de vista desde donde se puede abordar. El presente trabajo, en particular, asume la convivencia escolar como una consecuencia de la forma como se dirige una institución educativa, su identidad, la toma de decisiones, las interrelaciones, la cultura y los estilos comunicativos, entre otros (Marrugo et al., 2016). 
De ahí que todo aquel inmerso en el proceso de relaciones al interior de una comunidad educativa, tiene una responsabilidad y cumple un papel en el tipo de convivencia de esa institución (Megias, 2013).

Por tal razón, la convivencia escolar implica generar y respetar acuerdos y reglas compartidas, ya sean reglas implícitamente heredadas o reglas y acuerdos explícitamente establecidos, lo que a su vez involucra la tolerancia y confianza (Mockus \& Corzo, 2003). Por ende, es producto de las interacciones entre los sujetos que se dan en medio de las particularidades propias del contexto. Dichas interacciones "están mediadas por factores asociados a aspectos personales y colectivos de los distintos actores que conviven en el contexto educativo" (Camacho, Ordoñez, Roncancio \& Vaca, 2017: 26).

Por supuesto, son múltiples las causas por las que se puede ver afectada la convivencia escolar en las instituciones educativas. De acuerdo con el estudio realizado por López, Carvajal, Soto y Urrea, (2013), el cual evaluó la convivencia escolar en la capital y algunas zonas aledañas, evidenciando que, en los colegios de Bogotá, existen problemas como la agresión física y verbal, el acoso en sus diferentes manifestaciones y la falta de respeto e intolerancia entre pares y superiores. Esto lleva a concluir que la convivencia escolar está asociada a la forma como se solucionan los problemas, pues la "la violencia es independiente del tipo de establecimiento, edad, estrato y procedencia familiar (nuclear o no nuclear)" (López et al., 2013: 383).

A partir de esto, cobra sentido el trabajo de Chaux, Lleras y Velásquez (2004), quienes subrayan que la convivencia pacífica busca, a través del diálogo y la negociación, mediar ante los conflictos, para así manejarlos sin agresión y encontrar soluciones que beneficien a todas las partes, lo cual no implica la ausencia de conflictos. La convivencia escolar enfatiza en las relaciones que promueven una escucha abierta, vínculos de confianza y muestras de afecto positivo que benefician a todos los actores involucrados de manera pacífica (Marrugo et al., 2016).

\subsection{TRAYECTORIA FORMAL DE LA CONVIVENCIA EN COLOMBIA}

En Colombia, desde el cambio constitucional de 1991 la convivencia empezó a ser un tema relevante que debía estar a cargo de las instituciones de educación pública y privada, a través de las cátedras de instrucción cívica, las cuales, enmarcadas en los valores y la participación ciudadana formarían personas respetuosas de la ley (Constitución Política Colombiana, 1991, art.41).

En 1994, en la Ley general de Educación, la convivencia es incluida dentro de los objetivos generales de la educación, donde se promueve la comprensión de la realidad nacional a través del fortalecimiento de la convivencia social, la tolerancia y democracia como parte de los valores propios de nuestra nacionalidad. Además, se estipula el manual de convivencia como parte del proyecto educativo institucional, en el cual se deben incluir procedimientos para regular y resolver los conflictos (Ley 115, 1994).

Entre los años 2002 y 2006 se desarrolló el proceso de construcción de los Estándares Básicos de Competencias y con ellos se establecieron los referentes comunes para buscar una educación de calidad, a la que tienen derecho todos los ciudadanos independientemente de la región en la cual viven (MEN, 2013). Dicha educación no solo pretende que los estudiantes adquieran conocimientos acerca de asignaturas como matemáticas, lenguaje, ciencias sociales y naturales, sino también persigue la consolidación de una educación 
integral donde aprendan a convivir y puedan desempeñarse como ciudadanos activos de la sociedad. A través de los Estándares Básicos de Competencias Ciudadanas, el gobierno intentó lograr este último objetivo, convirtiéndolos en "una propuesta que le apuesta a la construcción de comunidades más pacíficas, democráticas, participativas, incluyentes y justas" (MEN, 2006:149).

Los estándares básicos de competencias ciudadanas se plantearon con el objetivo de fortalecer la participación democrática a través del desarrollo de aptitudes, las cuales demandan conocimientos básicos, habilidades comunicativas, cognitivas y emocionales propias de cada etapa de desarrollo (MEN, 2006). Es así, como la comunidad educativa experimentó una transición en la manera de entender la convivencia, pues se pasó de una instrucción tradicional a través de la materia cívica, a la introducción normalizada del tema en los manuales de convivencia, su inserción transversal en todas las asignaturas y el involucramiento de toda la comunidad educativa en su comprensión y mejoramiento.

Ya en el año 2013, la convivencia escolar la reglamentó el Sistema Nacional de Convivencia Escolar y Formación para el ejercicio de los Derechos Humanos, la Educación para la Sexualidad y la Prevención y Mitigación de la Violencia Escolar, con la Ley 1620 la cual formalizó y retomó la importancia de la convivencia escolar con el fin de formar ciudadanos que contribuyeran a la construcción de una sociedad democrática. De esta manera, el MEN (2013) propuso crear acciones para que todas las entidades involucradas en el sistema educativo fortalecieran el clima escolar a través de la ciudadanía activa y la convivencia pacífica. De acuerdo con Bocanegra y Herrera (2017), la ley pretendía:

Garantizar la existencia de una ruta de atención integral para los conflictos escolares; fomentar y fortalecer la educación en y para la paz; y garantizar los mecanismos de prevención, protección, detección temprana y denuncia de todas aquellas conductas que atenten contra la convivencia y los derechos de los miembros de la comunidad educativa, especialmente de los educandos (Bocanegra \& Herrera, 2017:199).

Esta ley hace énfasis entonces en la necesidad de comprender la convivencia escolar como un asunto colectivo cuya responsabilidad se comparte con toda la comunidad educativa y los demás sectores involucrados, la cual tiene como objetivo adoptar estrategias que promuevan y fortalezcan la mediación, la reconciliación y la divulgación de experiencias exitosas, generando estrategias pedagógicas que permitan articular los procesos de formación entre las distintas áreas de estudio (MEN, 2013).

\subsubsection{Experiencias sobre convivencia en Colombia}

Son varias las investigaciones hechas entorno a la convivencia en Colombia, algunas se refieren a estrategias que se pueden implementar para ayudar al mejoramiento de la convivencia en las instituciones educativas como el "Juego libre y convivencia escolar en el recreo de dos instituciones públicas de Bogotá. Una mirada desde la perspectiva del adolescente en el 2016" realizada por (Peña, Rodríguez \& Rojas, 2015), y "El Cuento infantil como estrategia pedagógica para fomentar la convivencia escolar en el aula multigrado" (Martínez, 2016) el cual utilizó el fortalecimiento de valores como estrategia para mejorar el ambiente escolar del aula. 
Otras investigaciones analizan los factores que influyen en ésta: problemáticas de convivencia escolar en las instituciones educativas del caribe colombiano: análisis desde la pedagogía social para la cultura de paz en el 2017; prevalencia del hostigamiento escolar en las instituciones públicas de Bucaramanga-Colombia de 2016; y prácticas agresivas en el aula, influidas por factores socioculturales y su relación con la construcción y desarrollo de la convivencia escolar (Pérez \& Pinzón, 2013). Lo que da cuenta que en el país hay una preocupación por el mejoramiento de la convivencia en los contextos educativos.

De igual forma, el distrito en cabeza de la Secretaría de Educación bienalmente hace un reconocimiento a aquellos colegios públicos que en la semana del "el colegio, la comunidad y la ciudad" visibilizan sus trabajos relacionados con el mejoramiento de la convivencia escolar. Para el año 2017 fueron seleccionados 25 establecimientos educativos que implementan estas iniciativas (Compartir palabra maestra, 2017).

\subsubsection{Convivencia escolar y competencias ciudadanas}

Las competencias ciudadanas son las habilidades y conocimientos que poseen las personas para relacionarse en los contextos a los cuales pertenecen, son metas tanto personales como estructurales que contribuyen a la construcción de la sociedad (MEN, 2006; Corporación Opción Legal, 2011). La formación en competencias ciudadanas se vuelve un proceso de aprendizaje para la vida, en el que no sólo el sujeto es partícipe, sino que implica la articulación de diferentes estrategias y prácticas que integren a la comunidad educativa en general (Ruiz \& Chaux, 2005).

El Ministerio de Educación (2006), definió tres ámbitos que organizan los estándares básicos de competencias ciudadanas y permite que los individuos desarrollen de manera integral y complementaria estas competencias. El primer ámbito es la Participación y responsabilidad democrática, abarca la importancia de saber convivir, respetando y aceptando la existencia de diferentes formas de pensar, expectativas y puntos de vista. En el segundo ámbito tiene como nombre, Convivencia y paz, se pretende generar intervenciones activas y críticas que comprometan acuerdos colectivos que afecten positivamente nuestro entorno. El último ámbito es la Pluralidad, identidad y valoración de las diferencias, el cual promueve la valoración y respeto de la diferencia e identidades particulares (MEN, 2006; COL, 2011).

Asimismo, la Ley 1620 de 2013 en el artículo 36 sobre las acciones del componente de promoción, propone la articulación de proyectos que promuevan el desarrollo de competencias ciudadanas para fortalecer la convivencia escolar. Las competencias ciudadanas dentro de los estándares básicos, se identificaron y organizaron de la siguiente forma:

- Las competencias comunicativas: necesarias para entablar una conversación se refieren a la escucha activa, asertividad y argumentación sin la intención de hacer daño alguno.

- Las competencias emocionales: se enfocan en la identificación y respuesta constructiva ante las emociones propias y las de los demás.

- Las competencias cognitivas: atañen al manejo que se le puede dar a la información recibida del contexto, es la toma de perspectiva, metacognición, pensamiento crítico y analítico, entre otros. 
- Las competencias integradoras: las cuales articulan todas las otras competencias, es decir para manejar conflictos pacíficamente es necesario tener unos conocimientos básicos, mirar y proponer estrategias para resolverlos, tener un manejo de las emociones y saber comunicarnos asertivamente (MEN, 2006; COL, 2011).

La articulación de las competencias ciudadanas implica retos significativos en el planteamiento e implementación del proceso de formación en ellas, pues es una construcción que se debe hacer conjuntamente con las entidades educativas, los docentes, los estudiantes y los padres de familia, utilizando diferentes estrategias que aporten a la transformación de la convivencia escolar y, con ella, al cambio social.

De esta manera, y no solo para dar cumplimiento a lo propuesto a la ley colombiana sino también para crear mejores ambientes escolares propicios para el aprendizaje, se hace necesario pensar en estrategias diferentes, adaptadas a las características culturales, sociales, cognitivas y emocionales de los estudiantes, por tanto, a continuación, se profundizará en una estrategia que ya está siendo empleada en varios contextos y la cual podría servir como una alternativa para fortalecer la convivencia escolar.

\section{MINDFULNESS}

El Mindfulness tiene diferentes traducciones y significados. Sin embargo, para fin de este artículo, se retomará la definición de uno de sus principales exponentes: Kabat-Zin. Este autor define el Mindfulness como la conciencia, una conciencia que se desarrolla prestando atención concreta en el momento presente, sostenida, deliberada y sin juicios de valor (Kabat-Zin, 2013). El concepto Mindfulness fue traducido por primera vez de la palabra Sati, proveniente de la lengua Pali (versión popular del sánscrito utilizada por Buda), por Thomas William Rhys Davis en 1881, en castellano este término traduce conciencia pura, meditación, toma de conciencia y el más utilizado: "atención plena” (Rivera, 2013). El concepto de Mindfulness tiene un origen budista y una relación con la meditación, específicamente la conexión de la mente y el cuerpo con el tiempo presente.

En Occidente, el Mindfulness y los temas relacionados con esta práctica, iniciaron en la década de los sesenta con el movimiento hippie (Sánchez, 2011). Ya en los años ochenta empezó a ser retomado por el área educativa, donde se abordó el tema de una educación Mindful en relación con la atención y el aprendizaje en particular en estudiantes de edad escolar, universitarios y adultos mayores (Langer \& Moldoveanu, 2000). Hacia los noventa e inicios del 2000 y a partir de los programas proporcionados por Kabat-Zin Mindfulness-Based Stress Reduction (MBSR), los estudios se enfocaron en el tratamiento de enfermedades crónicas como el estrés, el dolor, la ansiedad, entre otros. Según afirmó Proulx (2003) había más de 240 programas alrededor del mundo que aplicaban el MBSR para mitigar los efectos del estrés y diversas enfermedades crónicas.

En la Tabla 1 se resume la práctica del Mindfulness y las acciones a realizar en un periodo de ocho semanas, según lo propuesto por Kabat-Zin (2016) y Moreno (2015). 
Tabla 1. Programa 8 semanas de Mindfulness.

\begin{tabular}{|c|c|c|}
\hline Semana & Actividad & Tiempo estimado al día \\
\hline Semana 1 y 2 & $\begin{array}{l}\text { Escáner Corporal } \\
\text { Atención a la respiración }\end{array}$ & $\begin{array}{l}45 \text { minutos } \\
10 \text { minutos }\end{array}$ \\
\hline Semana 3 y 4 & $\begin{array}{l}\text { Atención a la respiración } \\
\text { Uno días Escáner corporal } \\
\text { Otros días Yoga atento-Hatha }\end{array}$ & $\begin{array}{l}15 \text { a } 20 \text { minutos } \\
45 \text { minutos }\end{array}$ \\
\hline Semana 5 y 6 & $\begin{array}{l}\text { Alternar Meditación sentado con Yoga de pie o Yoga atento } \\
\text { Paseo atento }\end{array}$ & 45 minutos \\
\hline Semana 7 & $\begin{array}{l}\text { Combinar en los días: } \\
\text { Escáner corporal } \\
\text { Meditaciones } \\
\text { Yoga }\end{array}$ & 45 minutos \\
\hline Semana 8 & $\begin{array}{l}\text { Escáner corporal } \\
\text { Meditaciones } \\
\text { Yoga } \\
\text { Paseo atento }\end{array}$ & 45 minutos \\
\hline
\end{tabular}

Nota: Cuadro realizado con base en la información de Kabat-Zin (2016) y Moreno (2015).

La propuesta de Kabat-Zin consiste en preparar el cuerpo y la mente durante 8 semanas para la atención plena, lo que significa ser consciente de cada instante. Lo anterior se logra prestando atención a todas las cosas que hacemos, pensamos y sentimos. Esta práctica conlleva a desarrollar el autocontrol desde la capacidad de relajarse, desarrollar la conciencia y teniendo una perspectiva más profunda de todas las cosas (Kabat-Zin, 2016).

Por otra parte, en relación con la psicoterapia, el Mindfulness ha sido una de las prácticas utilizadas por varias de las conocidas terapias de tercera generación, y aquellas que las anteceden, para modificar los problemas desde una mirada global y no solo comportamental. Además de utilizar nuevas técnicas de intervención como el Mindfulness, estas terapias intentan que el sujeto contemple los problemas desde una visión más amplia haciendo conciencia de ellos y modificándolos desde la aceptación (Santamaría \& Yela, 2016).

Algunas de las principales terapias que utilizan parte de los principios del Mindfulness son: El programa de Reducción del Estrés Basado en la Atención Plena (MBSR); la terapia cognitiva basada en la conciencia plena (MBCT); la Terapia de Aceptación y Compromiso (ACT); y la Terapia Conductual Dialéctica (DBT) utilizada en el tratamiento del trastorno límite de la personalidad y disminución de la conducta suicida (Brito, 2011; Santamaría y Yela, 2016). Cada uno de estos programas manejan un instructivo, organizado y multimodal, el cual se aplica para fomentar la conciencia plena en la focalización del presente.

De estos programas se han realizado diversos estudios sobre su eficacia y según Davidson et al. (2003) y Siegel (2007) la aplicación de estas terapias y los diferentes estudios realizados, dan cuenta que ser consciente del presente, mejora la función inmune, la satisfacción relacional e incluso mejora la sensación de claridad. El cambio cognitivo en 
la percepción de los pensamientos explica el efecto que tiene la atención plena (Linehan, 1993; Sarmiento \& Gómez, 2013) en las personas que reciben este tipo de intervenciones. Por esta razón, la práctica de Mindfulness, junto con la meditación, se constituyen como herramientas que favorecen el descentramiento del pensamiento, lo cual lleva a la persona a comprender que los pensamientos son solo eso y no reflejos incuestionables de la realidad (Sarmiento \& Gómez, 2013). A la larga, esto traerá como consecuencia una mejora en la forma como los sujetos se relacionan con el mundo.

\subsection{MINDFULNESS EN LA ESCUELA}

La escuela como uno de los contextos sociales relevantes para el desarrollo psicosocioafectivo de las personas, debe ser un lugar que promueva un entorno adecuado, un espacio en el cual los sujetos inmersos en el tengan la posibilidad de reflexionar sobre sus creencias, pensamientos, percepciones y conocimientos. La aplicación del Mindfulness en el contexto educativo surge, en parte, de la necesidad de hacerle frente a diferentes situaciones de estrés, ansiedad, convivencia y angustia que presentan los alumnos y docentes frente a las situaciones vividas diariamente.

El entrenamiento en Mindfulness en la escuela está dirigido a consolidar una conciencia plena basada en la respiración y en la coherencia interna que permita establecer una relación dialógica, base de un pensamiento creativo e interrelacional (Bai, Scott \& Donald, 2009). Esta práctica ayuda en la regulación de las emociones, en general y en situaciones críticas, lo que se traduce que el estudiante sea más empático y perciba el sufrimiento de los otros y responda a ello (Lantieri \& Zakrzewski, 2015).

Por otro lado, los estudios realizados por Langer y Moldoveanu (2000) con niños, estudiantes universitarios y ancianos, en la práctica de la atención plena, descubrieron que sí se instruye a las personas para que varíen el estímulo, es decir, para que no solo se focalicen en una cosa, sino que observen nuevas cosas al respecto, entonces la atención mejora; el resultado es un mayor gusto por la tarea y una mejoría en la memoria.

En países como México y España se están elaborando, desde grupos de investigación hasta proyectos en Mindfulness que incorporan variables como la inteligencia emocional y manejo del estrés en el abordaje del contexto educativo. Ejemplo de esto es el Programa de Educación Socio-Emocional (SEA), del grupo GROP de la Universidad de Zaragoza, el cual aborda no sólo problemas de afectividad y comportamiento en los estudiantes, sino que equilibra su atención e introspección en las actividades diarias, a través de la implementación del Mindfulness en sus talleres de habilidades para la vida (Urrego \& Castillo, 2015).

En Colombia, aunque la práctica es relativamente reciente, ya es posible encontrar algunos estudios y estrategias impartidas basadas en la práctica del Mindfulness. En relación con la práctica en el contexto educativo Alajmo y Jiménez (2016) encontraron que este programa mejoró la concentración asociada al trastorno con la regulación de pensamientos distractores en los participantes. Otro estudio encontrado en nuestro país en el contexto educativo es el de "Estrés escolar y empatía en estudiantes de bachillerato practicantes de Mindfulness" realizado en Bogotá con una metodología cuantitativa correlacional y el cual, después de dos meses de intervención, concluyó que la práctica del Mindfulness ayudó a disminuir el estrés escolar y a aumentar la empatía en los estudiantes participantes (Sierra, Urrego, Montenegro \& Castillo, 2015). 
Por último, a nivel teórico, podemos encontrar el artículo "Mindfulness y sus aplicaciones: las posibilidades en el contexto escolar", el cual hace un recuento sobre las investigaciones que se han realizado del tema en el mundo y en nuestro país, concluyendo que es una práctica incidente en el mejoramiento de los contextos educativos y por esto es necesario seguir ahondando e investigando en ella (Urrego \& Castillo, 2015).

En relación con las prácticas y estrategias concretas de Mindfulness que se han ido impartiendo en nuestro país, es clave mencionar el programa Respira, un programa pedagógico que busca llevar la práctica de Mindfulness (atención plena) a los colegios más afectados por la violencia, para fomentar el aprendizaje socio-emocional y el bienestar docente y estudiantil (Save The Children, 2018). Entre el 2014 y 2016, este programa, en asociación con la ONG Save The Children, se ha implementado en alrededor de 28 instituciones educativas públicas de Tumaco (Nariño), Tambo y Patía (Cauca), Cali, Medellín y Bogotá. De acuerdo a los análisis realizados por la Universidad de los Andes, en los participantes hubo una reducción de los síntomas de ansiedad y depresión, una mayor regulación emocional (particularmente de la ira), una reducción de la agresión reactiva y un aumento en la escucha activa (Respira, 2017).

En relación con las estrategias utilizadas en los escenarios educativos del distrito, se encontró una experiencia exitosa en el Colegio Rodrigo Lara Bonilla, de la localidad de Ciudad Bolívar. Desde el 2007, a través de la práctica del Mindfulness, en cabeza del profesor Alexander Rubio, se han logrado mejorar las relaciones entre pares, la concentración, las destrezas físicas y hasta el desempeño académico de los estudiantes participantes en su iniciativa (El yoga y otras nuevas formas de enseñar, 2018). Estas experiencias y los demás estudios encontrados en torno a la escuela y las necesidades que emergen de ella, dan cuenta de la importancia de seguir investigando sobre este contexto y sobre nuevas prácticas y estrategias que permitan a los docentes y a la comunidad educativa en general abordar aquellas necesidades de una manera asertiva en pro de una educación integral.

\section{CONSIDERACIONES FINALES}

Se puede percibir que tanto la convivencia escolar como el Mindfulness son temáticas que actualmente están en auge, en tanto son pertinentes para mejorar la realidad social de Colombia. A pesar de haber vivido un proceso de paz, Colombia sigue reportando diferentes problemáticas de intolerancia social, por ejemplo, la violencia al interior y alrededor de las instituciones educativas, la cual va en aumento. Lo que exige cambios en las prioridades de la escuela, como en la formación de valores y la capacitación de los estudiantes en competencias para la vida (COL, 2011). Además de aparecer la necesidad de fomentar espacios que permitan que los estudiantes desaprendan las conductas violentas a través de la implementación de nuevas alternativas que generen un aprendizaje significativo en las relaciones que los estudiantes establecen dentro del contexto educativo (Buritica-Morales \& Nañez-Rodríguez, 2017).

Frente a esto, son varios los esfuerzos que los diferentes entes educativos, en cabeza del Ministerio de Educación han desarrollado para continuar en la transformación de la educación de nuestro país. La creación de los Estándares Básicos de Competencias, la encuesta de percepción de la convivencia escolar en 2006 y la implementación de la Ley 1620, entre otros micro procesos que se han dado en muchas de las instituciones 
educativas y que son muestra de los esfuerzos realizados para fortalecer la situación de la convivencia escolar.

Sin embargo, revisando varios de estos abordajes se evidencia un mayor énfasis en las acciones de atención, las cuales buscan intervenir los comportamientos riesgosos para la materialización efectiva de los DDHH, Sexuales y Reproductivos en la escuela, evitando que se constituyan en patrones de interacción que alteren la convivencia de los miembros de la comunidad educativa (ley 1620 art. 37). Las propuestas para mejorar la convivencia escolar dan prioridad a los protocolos a seguir frente a una situación ya ocurrida, y en otros casos al fortalecimiento de las competencias ciudadanas comunicativas, acerca de cómo dialogar con un compañero y los pasos para resolver un conflicto entre pares. No obstante, dejan de lado lo que este articulo pretende resaltar en cuanto a la práctica del Mindfulness y la convivencia como lo son: las experiencias internas, la construcción personal, el desarrollo y fortalecimiento de los pensamientos, sensaciones y niveles de concentración de cada uno de los estudiantes.

En ese sentido, el Mindfulness es una herramienta que complementa estos procesos, pues ayuda a los estudiantes a tomar conciencia e identificar la conexión entre sus emociones, pensamientos y sensaciones corporales. Lo que los hace capaces de regular sus emociones, los lleva a mejorar su comportamiento, niveles de estrés, relaciones y capacidad de concentración. Es así como las prácticas de atención plena conectan las experiencias internas y externas de los estudiantes y les ayudan a ver la congruencia entre las mismas, (Lantieri \& Zakrzewski, 2015).

En cambio, la convivencia escolar al ser consecuencia de las relaciones e interacciones de las experiencias externas $y$, en este escrito particularmente, consecuencia de las estrategias implementadas por el MEN para el fortalecimiento de las competencias ciudadanas, lo que la lleva a ser una vivencia producto de las acciones institucionalizadas en la Ley de Educación. Así, en la cartilla de orientaciones para la institucionalización de las competencias ciudadanas publicada por el MEN en el 2011, se puede identificar el énfasis que se le da a la convivencia desde la prevención de la violencia escolar, calificando ésta como el antónimo de la Convivencia escolar, es decir, asumiendo entonces, que si se mitiga este problema desde diferentes áreas: manual de convivencia, gobierno escolar, comité de convivencia, prácticas curriculares, gestión administrativa, participación estudiantil y en general cultural institucional, se logrará el mejoramiento de la convivencia escolar, dejando de lado otros factores por los cuales ésta se puede ver afectada.

En este sentido y como fue objetivo de este artículo la convivencia debe también tener un proceder interno en cada sujeto, quien primero debe identificar sus habilidades, pensamientos y emociones, lo cual se puede conseguir a través de la práctica del Mindfulness que funciona de adentro hacia afuera, direccionando la capacidad de cada sujeto para desarrollar sus cualidades y por ende relacionarse de una forma más asertiva y empática (Lantieri \& Zakrzewski, 2015). Con el apoyo de los docentes y las nuevas estrategias que se están implementando en las instituciones educativas, como son los proyectos transversales que varias de ellas ya han incorporado dentro de sus planes curriculares, se puede promover un fortalecimiento de las competencias ciudadanas y el desarrollo de una educación de calidad dentro de ambientes escolares mejores.

Finalmente, se concluye que de acuerdo a la evidencia existente hasta el momento sobre los beneficios de la práctica del Mindfulness, se considera relevante seguir implementando esta práctica en los contextos educativos y así a futuro, el sistema educativo promover 
la inclusión del Mindfulness como un recurso psicosocioemocional dirigido no solo a los estudiantes, sino a docentes, directivos y padres de familia, que ayude a mejorar el desempeño personal, emocional, cognitivo y académico de los mismos.

\section{REFERENCIAS BIBLIOGRÁFICAS}

Alajmo, T. \& Jiménez, L. (13 de junio de 2016). Mindfulness y el manejo de ansiedad en niños escolarizados, Evaluación del efecto de un programa basado en Mindfulness para el manejo de ansiedad en niños escolarizados. [Mensaje en un blog]. Compartir Palabra Maestra. Recuperado desde https://compartirpalabramaestra.org/alianza-gimnasio-campestre-compartir/mindfulnessy-el-manejo-de-ansiedad-en-ninos-escolarizados

Bai, H., Scott, C. \& Donald, B. (2009). Contemplative pedagogy and revitalization of teacher education. Alberta Journal of Educational Research, 55(3), 319-334. Recuperado el 4 de febrero de 2018 desde http://summit.sfu.ca/item/13208

Barragán, R., Lewis, S. \& Palacio, J. (2007). Autopercepción de cambios en los déficit atencionales intermedios de estudiantes universitarios de Barranquilla sometidos al Método de Autocontrol de la Atención (Mindfulness). Revista Salud Uninorte, 23(2), 184-192. Recuperado el 10 de febrero de 2018 desde http://rcientificas.uninorte.edu.co/index.php/salud/article/view/4032/2468

Bocanegra, H. \& Herrera, C. (2017). La ley 1620 de 2013 y la política pública educativa de convivencia escolar en Colombia: entre la formalidad jurídica y la realidad social. Revista Republicana, 23, 185-214. DOI http://dx.DOI.org/10.21017

Brito, G. (2011). Programa de reducción del estrés basado en la atención plena (Mindfulness): sistematización de una experiencia de su aplicación en un hospital público semi-rural del sur de chile. Psicoperspectivas, 10(1), 221-242. DOI:10.5027/PSICOPERSPECTIVAS-VOL10ISSUE1-FULLTEXT-121

Buritica-Morales, D. \& Nañez-Rodríguez, J. (2017). El recreo: Un camino hacia la prevención de comportamientos agresivos. Revista Inclusión \& Desarrollo, 4(1), 11-35. DOI: http://dx.DOI. org/10.26620/uniminuto.inclusion.4.2.2017.29-39

Camacho, N., Ordoñez, J., Roncancio, M. \& Vaca, P. (2017). Convivencia escolar y cotidianidad: una mirada desde la inteligencia emocional. Revista Educación y Desarrollo Social, 11(1) 24-47. DOI org/10/18359/reds.2649

Chaux, E., Lleras, J. \& Velásquez, A.M. (Eds.). (2004). Competencias ciudadanas: de los estándares al aula. Una propuesta integral para todas las áreas académicas. Bogotá: Ministerio de Educación Nacional y Universidad de los Andes.

Compartir palabra maestra (2017). Bogotá reconoce a los mejores proyectos de convivencia escolar. Bogotá, Colombia. Recuperado el 5 de febrero de 2018 desde https://compartirpalabramaestra. org/actualidad/noticias/bogota-reconoce-los-mejores-proyectos-de-convivencia-escolar

Constitución política colombiana (1991). Asamblea Nacional Constituyente, Bogotá, Colombia, 6 de Julio de 1991.

Corporación Opción Legal. (2011). El Desarrollo de Competencias Ciudadanas en la Escuela Módulo: "Resiliencia y competencias ciudadanas”. Bogotá: Opciones Gráficas Editores Ltda.

Davidson, RJ., Kabat-Zinn, J., Schumacher, J., et al. (2003). Alterations in brain and immune function produced by Mindfulness meditation. Psychosomatic Medicine, 65(4), 564-70. DOI: http://dx.DOI.org/10.1097/00006842-200401000-00022

El yoga y otras nuevas formas de enseñar. (2018, 22 de enero). Revista Semana. Recuperado el 10 de febrero de 2018 desde https://www.semana.com/educacion/articulo/nuevas-alternativas-deensenanza-que-se-crean-en-el-aula-yoga/554485

Kabat-Zin, J. (2016). Vivir con plenitud las crisis, cómo utilizar la sabiduría del cuerpo y de la mente 
para enfrentarnos al estrés, dolor y la enfermedad. Barcelona: Editorial Kairos.

(2013). Mindfulness para principiantes. Barcelona: Editorial Kairós.

Herrera, B. \& Silas, J. (2017). Una forma de educación alternativa y el papel de los educadores como agentes "transformadores" con jóvenes en situación de riesgo. Sinéctica, (49), 1-19. Recuperado el 15 de febrero de 2018 desde http://www.scielo.org.mx/scielo.php?script=sci_ arttext\&pid=S1665-109X2017000200012\&lng=es\&tlng=es.

Hervás, G., Cebolla, A. \& Soler, J. (2016). Intervenciones psicológicas basadas en Mindfulness y sus beneficios: estado actual de la cuestión. Clínica y Salud, 27(3), 115-124. DOI: https://dx.DOI. org/10.1016/j.clysa.2016.09.002

Langer, E. \& Moldoveanu, M. (2000). The Construct of Mindfulness. Journal of Social Issues, 56(1), 1-9. DOI: https://DOI.org/10.1111/0022-4537.00148

Lantieri L. \& Zakrzewski V. (2015). How SEL and Mindfulness Can Work Together. Greater Good Magazine Science-Based Insights for a Meaningful Life. Recuperado el 1 de febrero de 2018 desde http://greatergood.berkeley.edu/article/item/how_social_emotional_learning_and_ Mindfulness_can_w

Ley 115. Congreso de la República de Colombia, Santa Fe de Bogotá, Colombia. 8 de febrero de 1994. Recuperado el 2 de febrero de 2018 desde http://www.mineducacion.gov.co/1621/ articles-85906_archivo_pdf.pdf

Ley 1620. Congreso de la República de Colombia, Santa Fe de Bogotá, Colombia. 15 de marzo de 2013. Recuperado el 3 de febrero de 2018 desde https://www.mineducacion.gov.co/1759/ articles-327397_archivo_pdf_proyecto_decreto.pdf

Linehan, M. (1993). Cognitive-behavioural treatmentof borderline disorder. New York: Guilford Press

López, C., Carvajal, C., Soto, M. \& Urrea, P. (2013). Factores asociados a la convivencia escolar en adolescentes. Educación y Educadores, 16 (3). Recuperado el 8 de febrero de 2018 desde http:// educacionyeducadores.unisabana.edu.co/index.php/eye/article/view/2716/3349

Maturana, H. (2002). Transformación en la convivencia (2nd Ed.). Santiago de Chile: Dolmen Ediciones S.A.

Marrugo, G., Gutiérrez, J., Concepción, I. \& Concepción, M. (2016). Estrategia de Convivencia Escolar Para la Formación de Jóvenes Mediadores de Conflictos. Escenarios, 14(1), 72-84. DOI: http://dx.DOI.org/10.15665/esc.v14i1.879

Martínez, M. (2016). El cuento infantil como estrategia pedagógica para fomentar la convivencia escolar en el aula multigrado. (Tesis de Pregrado). Corporación Universitaria Minuto de Dios, Bogotá, Colombia.

Megías, M. (2011). La convivencia escolar y cómo resolver los conflictos y la violencia en el aula. Granada: ADICE.

Ministerio de Educación Nacional. (2013). Guías pedagógicas para la convivencia escolar, Ley 1620 de 2013 - Decreto 1965 de 2013. Bogotá: MEN.

. (2011). Orientaciones para la institucionalización de las competencias ciudadanas, Cartilla 2 Mapa Programa de competencias ciudadanas. Bogotá: Amado Impresores S.A.S.

(2006). Estándares Básicos de Competencias en Lenguaje, Matemáticas, Ciencias y Ciudadanas, Guía sobre lo que los estudiantes deben saber y saber hacer con lo que aprenden. Recuperado el 5 febrero de 2018 desde https://www.mineducacion.gov.co/1621/articles-340021_ recurso_1.pdf

Mockus, A., y Corzo, J. (2003). Cumplir para convivir. Factores de convivencia y su relación con Normas y Acuerdos. Bogotá: Universidad Nacional de Colombia Unibiblos.

Moreno, N. (2015). Programa de Mindfulness de 8 semanas de Jon Kabat-Zinn. [Mensaje en un blog]. Nombre del blog. Recuperado el 16 de febrero de 2018 desde http://www. nicolasmorenopsicologo.com/psicologos-granada/programa-de-Mindfulness-de-8-semanas-dejon-kabat-zinn/ 
Peña, C., Rodríguez, L. y Rojas, B. (2015). Juego libre y convivencia escolar en el recreo de dos instituciones públicas de Bogotá: una mirada desde la perspectiva del adolescente, (tesis de Maestría). Universidad de la Salle, Bogotá, Colombia.

Pérez, N. y Pinzón, V. (2013). Prácticas agresivas en el aula, influidas por factores socioculturales y su relación con la construcción y desarrollo de la convivencia escolar. Estudio en los jóvenes del Grado Octavo de la Institución Educativa Corporación Colegio Bolivariano del Norte de la Ciudad de San José de Cúcuta. (Tesis de pregrado), Universidad nacional abierta y a distancia, Medellín, Colombia.

Proulx, K. (2003). Integrating Mindfulness-Based Stress Reduction. Holistic Nursing Practice, 17(4), 201-208. DOI: https://DOI.org/10.1111/0022-4537.00148.

Respira, (2017). Mindfulness para la Convivencia Escolar. Bogotá, Colombia. Recuperado el 8 de febrero de 2018 desde https://respira.co/respira_en_educacion https://respira.co/front/uploads/ informeanual20162.pdf

Rivera, F. (2013). Efecto de un programa de entrenamiento en Mindfulness en el desarrollo de habilidades psicoterapéuticas. (Tesis de maestría). Universidad de Chile, Santiago de Chile, Chile.

Ruiz-Silva, A. y Chaux, E. (2005). La formación de competencias ciudadanas. Bogotá: Asociación Colombiana de Facultades de Educación ASCOFADE.

Sánchez, G., (2011). Meditación, Mindfulness y sus efectos biopsicosociales, revisión de literatura. Revista electrónica de psicología, 14(2), 223-254.

Santamaría, C. \& Yela, J. (2016). Programa de reducción de estrés basado en Mindfulness y su aplicación al ámbito laboral. (Tesis de pregrado), Universidad Pontificia de Salamanca, Salamanca, España.

Sarmiento, M. \& Gómez, A. (2013). Mindfulness Una propuesta de aplicación en rehabilitación neuropsicológica. Avances en Psicología Latinoamericana, 31(1), 140-155. Recuperado el 17 de febrero de 2018 desde https://revistas.urosario.edu.co/index.php/apl/article/view/2368

Save the Children. (2018). Proyecto Respira. Bogotá, Colombia: Save the Children. Recuperado el 4 de febrero de 2018 desde https://www.savethechildren.org.co/proyecto-respira

Siegel, D. J. (2007). Mindfulness training and neural integration: differentiation of distinct streams of awareness and the cultivation of well-being. Social Cognitive and Affective Neuroscience, 2(4), 259-263. DOI: http://DOI.org/10.1093/scan/nsm034.

Sierra, O. et al. (2015). Estrés escolar y empatía en estudiantes de bachillerato practicantes de Mindfulness. Cuadernos de Lingüística Hispánica, 26, 175-197. Recuperado el 6 de febrero de 2018 desde https://revistas.uptc.edu.co/index.php/linguistica_hispanica/article/view/3685/3246

Urrego, G. \& Castillo, O. (2015). Mindfulness y sus aplicaciones: las posibilidades en el contexto escolar. Revista Dialéctica Libertadora, 8(2), 60-73. Recuperado el 6 de febrero de 2018 desde http://190.242.99.229/index.php/DialecticaLibertadora/article/view/591/558 